\title{
The OGLE-III catalog of variable stars: First results
}

\author{
Igor Soszyński ${ }^{1}$ \\ ${ }^{1}$ Warsaw University Observatory, Al. Ujazdowskie 4, 00-478 Warszawa, Poland \\ email: soszynsk@astrouw.edu.pl
}

\begin{abstract}
The third phase of the Optical Gravitational Lensing Experiment (OGLE-III) has been conducted since 2001 and regularly monitors the brightness of about 200 million stars. The OGLE-III fields cover both Magellanic Clouds and a large area in the Galactic bulge and disk. Here we describe the first parts of the OGLE-III Catalog of Variable Stars which is being prepared on the basis of these data. We present the principles of the catalog and methods used to select variable stars. We expect that the whole catalog will contain at least one million variable stars of all types. The catalog includes the list of variable sources along with their basic parameters, high precision multi-epoch $I$ and $V$-band photometry and accurate astrometry. All objects are classified and cross-identified with previously published catalogs. We also carry out a preliminary statistical analysis of these huge samples of variable stars.
\end{abstract}

Keywords. catalogs, Cepheids, stars: variables: other, Magellanic Clouds

\section{Introduction}

The Optical Gravitational Lensing Experiment is a long-term sky survey being conducted since 1992. One of the main scientific goals of the project is the searching for gravitational microlensing events (Paczyński 1986), but a huge amount of high quality photometric data collected by the OGLE survey are ideal material for many other astrophysical purposes. One of the most important results produced by the OGLE project were large catalogs of variable stars in the Magellanic Clouds and in the Galactic bulge.

The first phase of the OGLE project (OGLE-I) was conducted between 1992 and 1995 on the 1-m Swope telescope at Las Campanas Observatory in Chile. About 2 million stars were regularly monitored. This part of the survey resulted in catalogs of variable stars toward the Galactic bulge (Udalski et al. 1994), globular clusters $\omega$ Cen (Kałużny et al. 1996) and 47 Tuc (Kałużny et al. 1998), dwarf galaxies Sculptor (Kałużny et al. 1995) and Sagittarius (Mateo et al. 1995). In total, several thousands variables were identified on the basis of the OGLE-I observations.

In January 1997 the OGLE survey entered its second phase (OGLE-II). The upgrade included a new 1.3-m Warsaw Telescope at Las Campanas Observatory, dedicated to the project. The telescope was equipped with a "first generation" $2048 \times 2048$ CCD camera working in drift-scan mode. Comparing to the previous stage the observing capabilities of the project were increased by a factor of 30. The Magellanic Clouds were added to the list of regularly observed fields.

The outcome of variable stars increased significantly in comparison with the OGLE-I project. Thousands of Cepheids, RR Lyr stars, eclipsing binaries and long-period variables were detected in the Magellanic Clouds and Galactic bulge. Table 1 list the most important catalogs of variable stars published on the basis of the OGLE-II data. These samples often contained the largest sets of particular variable stars detected so far in any environment. 
Table 1. The OGLE-II catalogs of variable stars.

\begin{tabular}{|c|c|c|c|}
\hline Type of variable stars & Environment & $\begin{array}{l}\text { Number } \\
\text { of stars }\end{array}$ & Papers \\
\hline \multirow{2}{*}{ Classical Cepheids } & LMC & $1335+81$ & $\begin{array}{l}\text { Udalski et al. }(1999 \mathrm{~b}) \\
\text { Soszyński et al. }(2000)\end{array}$ \\
\hline & SMC & $2049+95$ & $\begin{array}{l}\text { Udalski et al. }(1999 \mathrm{c}) \\
\text { Udalski et al. }(1999 \mathrm{a})\end{array}$ \\
\hline Type II Cepheids & Bulge, LMC & 54,14 & Kubiak \& Udalski (2003) \\
\hline \multirow{3}{*}{ RR Lyrae } & LMC & 7612 & Soszyński et al. (2003) \\
\hline & $\mathrm{SMC}$ & 571 & Soszyński et al. (2002) \\
\hline & Bulge & 2700 & Mizerski (2003) \\
\hline \multirow{2}{*}{ Eclipsing Binaries } & LMC & 2580 & Wyrzykowski et al. (2003) \\
\hline & SMC & 1350 & Wyrzykowski et al. (2004) \\
\hline Miras and SRV & LMC & 3221 & Soszyński et al. (2005) \\
\hline$\delta$ Scuti & Bulge & 193 & Pigulski et al. (2006) \\
\hline$\beta$ Cephei, SPB & LMC, SMC & $98+90$ & Kołaczkowski et al. (2006) \\
\hline all & LMC, SMC & 68000 & Żebruń et al. (2001) \\
\hline all & Bulge & 200000 & Woźniak et al. (2002) \\
\hline
\end{tabular}

In 2000 the OGLE-II photometry was reprocessed using a newly developed Difference Image Analysis (DIA, Alard \& Lupton 1998; Woźniak 2000). The new method of the data reduction significantly increased the quality of the photometry and opened new possibilities for the detection of variable stars. The direct result of this analysis was the preparation of the huge catalogs of variable sources in the Magellanic Clouds (Żebruń et al. 2001) and Galactic bulge (Woźniak et al. 2002).

The next upgrade of the OGLE project came in 2001. The Warsaw Telescope was equipped with a new mosaic camera consisting of eight $2048 \times 4096$ chips which increased our sky coverage by an order of magnitude. The OGLE-III project announced many important discoveries including extra-solar planets detected using two methods: transits and microlensing events. Variable stars were also analyzed using OGLE-III data, however usually as a supplement of the OGLE-II photometry (the exception are planetary transits and microlensing events which obviously also are variable stars). In this paper we describe the new sub-project being conducted with the OGLE-III data - the OGLE-III Catalog of Variable Stars (OIII-CVS).

\section{OGLE-III data}

OGLE-III regularly monitors the brightness of about 200 million stars in 170 square degrees of the sky. To date more than 215000 frames have been collected which occupy about 25 TB of disk space. On average, several hundred photometric measurements per star have been secured, most of them in the standard $I$ band. About $10 \%$ of observations were obtained with the $V$ filter. All these data will be used for the selection and analysis of variable stars in the OGLE-III fields. Taking into account the size of the OGLE-II catalogs we expect that the number of variables detected in the OGLE-III fields will reach one million objects.

To search for variable sources we use the OGLE-III photometric data finally reduced by Udalski et al. (2008a). Comparing to the previous, provisional reductions, the new 

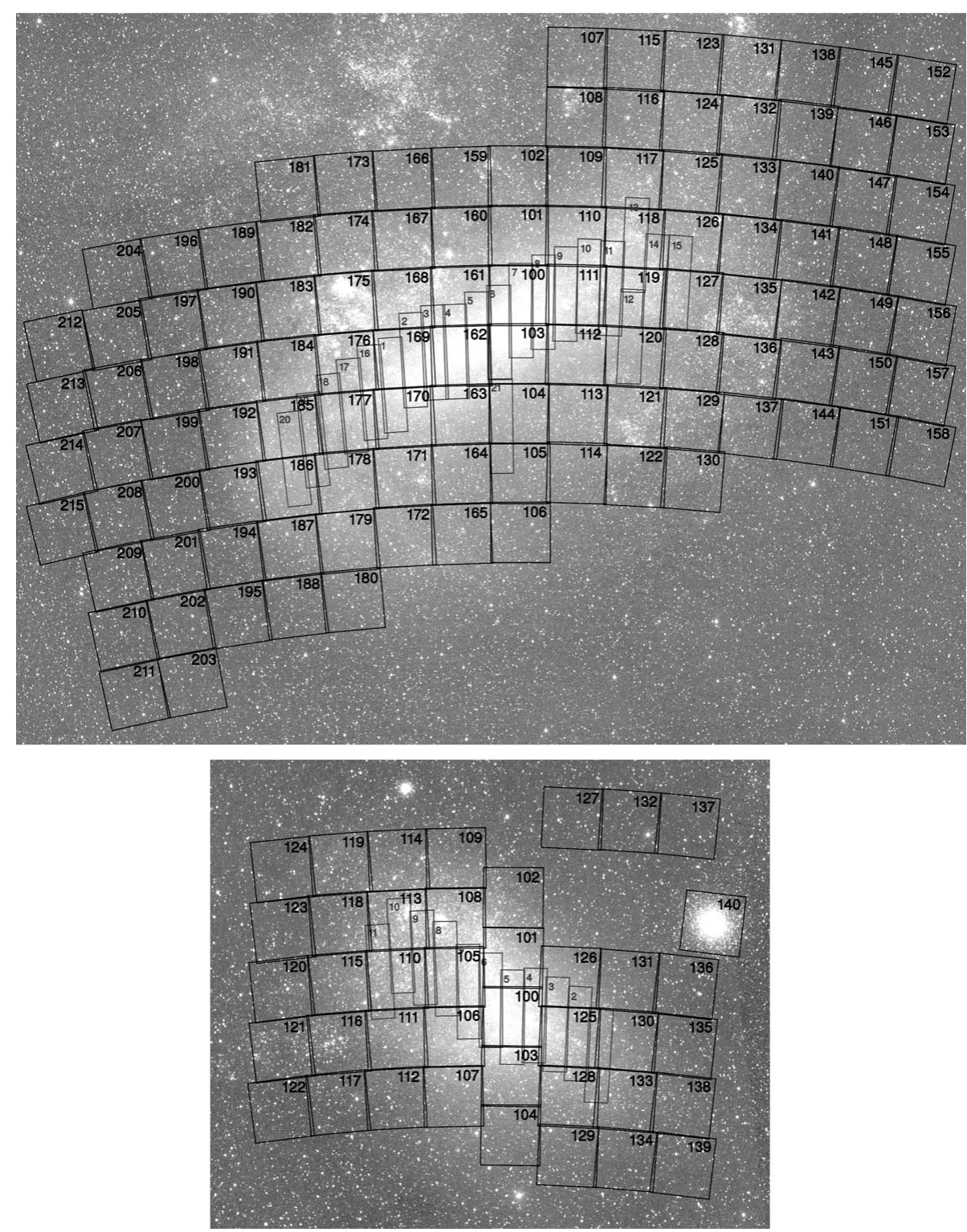

Figure 1. Contours of the OGLE-II (grey) and OGLE-III (black) fields in the LMC (upper panel) and in the SMC (lower panel) overplotted on the ASAS pictures.

data set is more accurate and well calibrated. The typical uncertainty of the photometric calibration is less than $0.02 \mathrm{mag}$. Moreover, even the gaps between the chips of the CCD mosaic are covered by our reductions, because, due to imperfections of the telescope pointing, the regions between the chips are also observed from time to time.

The OGLE-III fields cover about 40 square degrees of the densest regions in the LMC and about 14 square degrees in the SMC, including globular cluster 47 Tuc. Fig. 1 shows the contours of the OGLE-III fields plotted over pictures taken by the ASAS survey (Pojmański 1997). Very recently the OGLE-III photometric maps of the LMC were published by Udalski et al. (2008b). The maps contain precisely measured mean $I$ and $V$ 


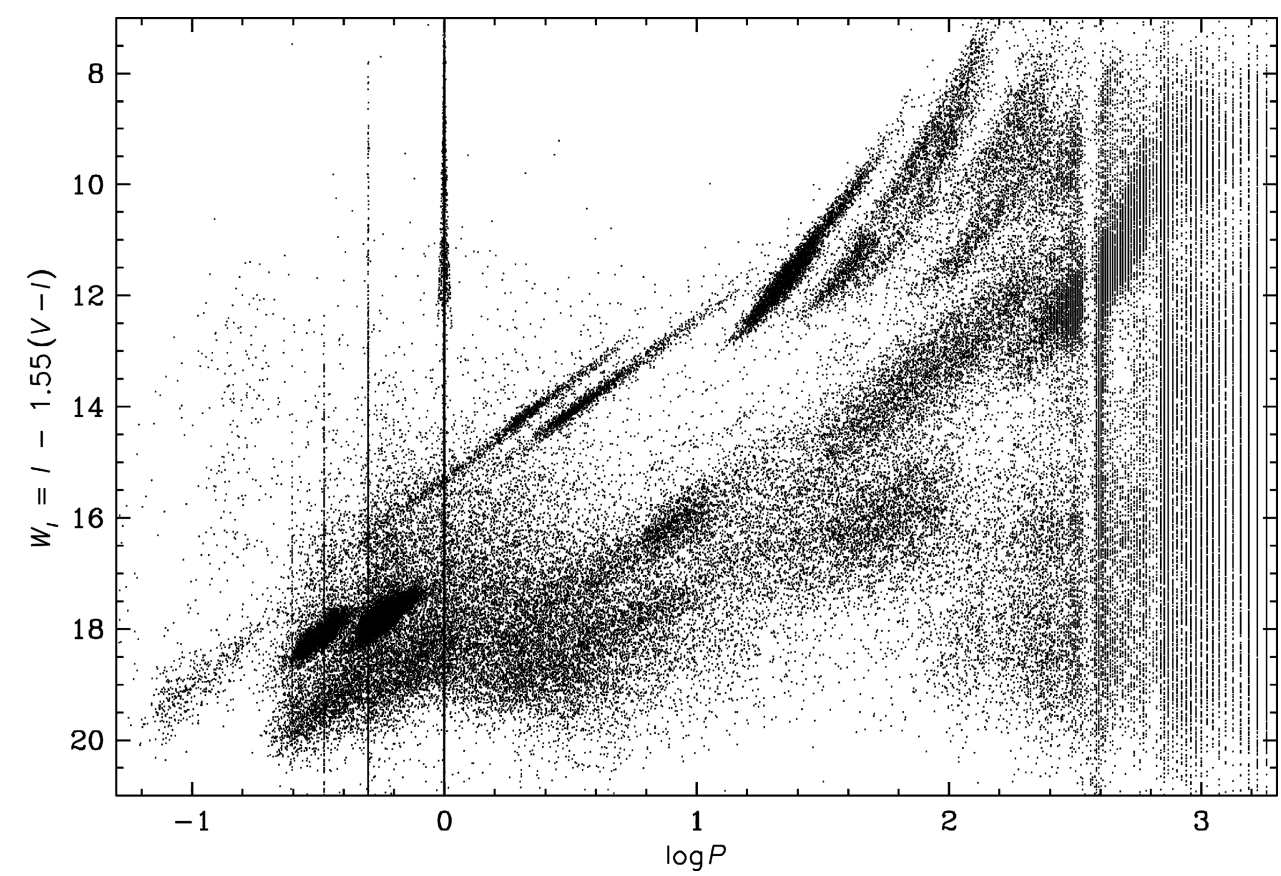

Figure 2. Period-luminosity diagram for the stars in the LMC with periodic signal-to-noise ratio larger than 7 .

magnitudes of about 35 million stars. The photometric maps of the SMC will be released very soon.

\section{The principles of the catalog}

The principles of the OIII-CVS are as follows. We plan to detect all variables sources in the OGLE-III fields. The catalog will be divided into parts consisting of different types of variable stars in different environments (LMC, SMC and Galactic bulge). Each piece of the catalog will be successively published in the electronic form only in the OGLE Internet Archive. The catalog data set will include VI multi-epoch photometry, basic parameters of the stars (coordinates, periods, mean magnitudes, amplitudes, parameters of the Fourier light curves decompositions), and $60^{\prime \prime} \times 60^{\prime \prime}$ finding charts.

All objects will be cross-identified with previously published samples of variable stars. Special attention will be paid to the completeness of the catalog. All previously known objects of the given type not present in our sample, will be carefully studied to find the reason of the absence. Finally, the catalog will not be a closed structure. For example, the photometry will be supplemented by new observations until the end of the OGLE-III project. Similarly, every new variable star of the given type found after the publication of the catalog will be added to the released list of objects.

All parts of the catalog will be accompanied by papers describing the methods of selection and analysis of the variable stars, showing objects of particular interest and preliminary statistical analysis.

\section{Selection of variable stars}

Selection and analysis of such a huge sample of variable stars obviously demands unprecedented efforts. Different types of variable stars will be searched using different 

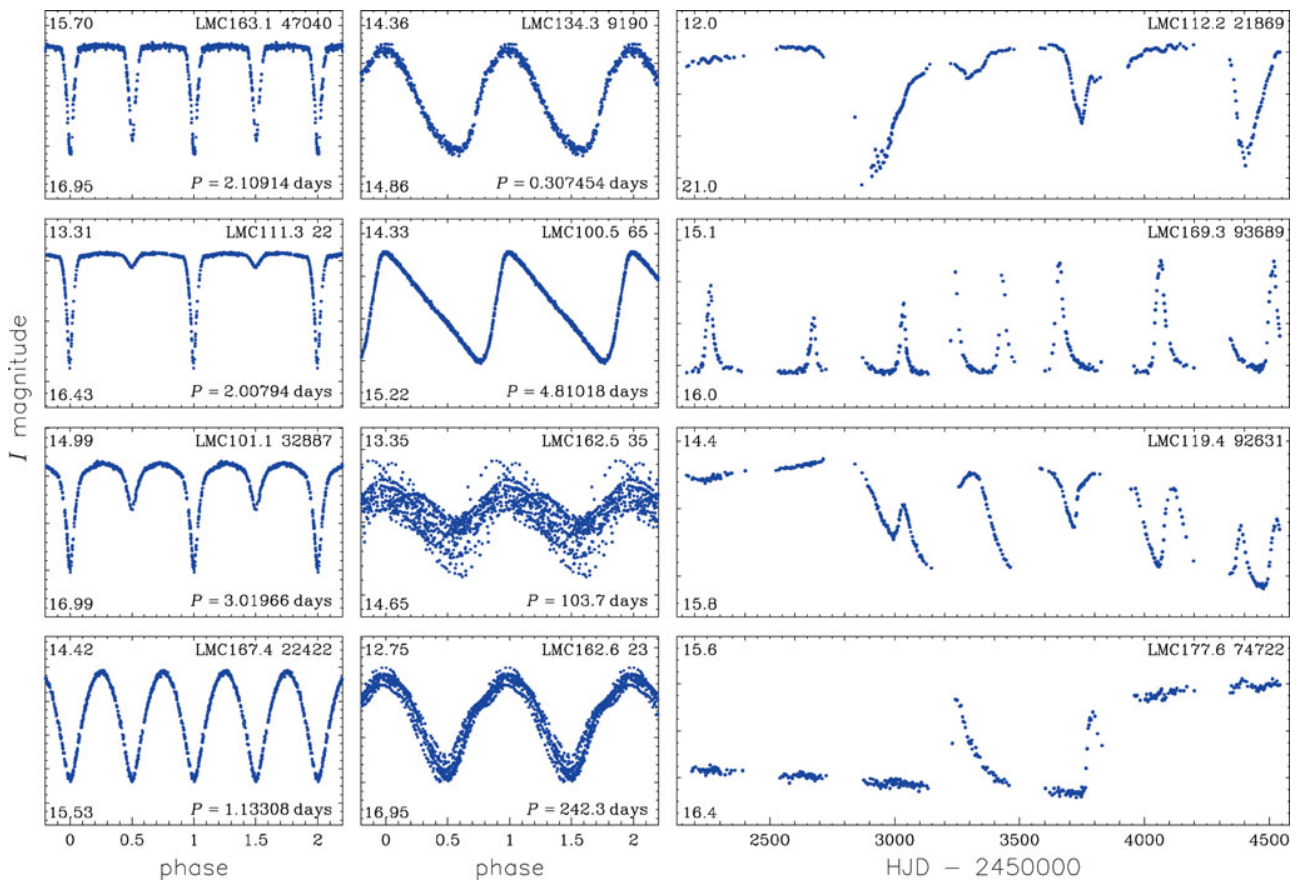

Figure 3. Exemplary light curves of variable stars in the LMC: eclipsing binary systems (left column), pulsating variables (middle column) and irregular variables (right column).

methods. To search for periodic variables, including very low amplitude and very faint stars, we conducted massive period search using supercomputers at the Interdisciplinary Centre for Mathematical and Computational Modelling (ICM). To date we derived periods for all 35 million stars in the LMC.

The results of these computations are shown in Fig. 2. We plotted here the extinction insensitive Wesenheit index $W_{I}=I-1.55(V-I)$ versus logarithm of the period (in days) for the stars in the LMC with the periodic signal-to-noise ratios larger than 7 . About 140000 of 35 million stars in the LMC are presented on this raw diagram. Apart from the stars with artificial periods equal to about $1,1 / 2,1 / 3$ or $1 / 4$ days, one can easily recognize a series of certain period-luminosity $(\mathrm{PL})$ relations for pulsating variables and binary systems. We draw the reader's attention to the sequences of classical Cepheids which are extended toward shorter periods and transform to High Amplitude $\delta$ Sct stars. Below this formation one can notice tens of thousands of RR Lyr stars. In the upper right corner of the diagram a large number of long-period variables follow a series of PL sequences. Finally, close binary systems - eclipsing and ellipsoidal variables — also delineate wide PL relations below Cepheids and long-period variables.

Fig. 3 shows the exemplary light curves of variable stars in the LMC. We present here periodic and non-periodic stars of various types.

\section{First results}

At present, the first part of the OIII-CVS has already been released (Soszyński et al. 2008b). It presents 3361 classical Cepheids in the LMC. The second part - type II Cepheids and anomalous Cepheids in the LMC - is in preparation.

Among large samples of variable stars very rare or even previously unknown types of objects can be found. For example studying classical Cepheids in the LMC we discovered 
two double-mode Cepheids of a new type — with the first and the third overtones simultaneously excited (Soszyński et al. 2008a). Besides, we identified three new triple-mode Cepheids, new classical Cepheids in eclipsing binary systems, including a system of two pulsators revealing eclipses, many Blazhko Cepheids and single-mode second-overtone Cepheids (Soszyński et al. 2008b).

\section{Summary}

The OGLE-III photometric database is a gold mine of information about stellar variability. Our ambitious plan to catalog all variable sources in the OGLE-III fields will be continued over the next several years. We expect that the final catalog will contain the largest number of variable stars detected so far by any large sky survey. First results show that among the large number of variable stars very uncommon or even new type of objects are present. Huge samples open new possibilities for statistical analysis of variable stars.

\section{Acknowledgements}

This work has been supported by Foundation for Polish Science through the Homing (Powroty) Program and by MNiSW grant NN203293533. The massive period searching was performed at the Interdisciplinary Centre for Mathematical and Computational Modelling (ICM).

\section{References}

Alard, C., \& Lupton, R.H. 1998, ApJ, 503, 325

Kałużny, J., Kubiak, M., Szymański, M., et al. 1995, A\&AS, 112, 407

Kałużny, J., Kubiak, M., Szymański, M., et al. 1996, A\&AS, 120, 139

Kałużny, J., Kubiak, M., Szymański, M., et al. 1998, A\&AS, 128, 19

Kołaczkowski, Z., Pigulski, A., Soszyński, I., et al. 2006, MemSAI, 77, 336

Kubiak, M., \& Udalski, A. 2003, AcA, 53, 117

Mateo, M., Udalski, A., Szymański, M., et al. 1995, AJ, 109, 588

Mizerski, T. 2003, AcA, 53, 307

Paczyński, B. 1986, ApJ, 304, 1

Pigulski, A., Kołaczkowski, Z., Ramza, T., \& Narwid, A. 2006, MemSAI, 77, 223

Pojmański, G. 1997, AcA, 47, 467

Soszyński, I., Udalski, A., Szymański, M., et al. 2000, AcA, 50, 451

Soszyński, I., Udalski, A., Szymański, M., et al. 2002, AcA, 52, 369

Soszyński, I., Udalski, A., Szymański, M., et al. 2003, AcA, 53, 93

Soszyński, I., Udalski, A., Kubiak, M., et al. 2005, AcA, 55, 331

Soszyński, I., Poleski, R., Udalski, A., et al. 2008a, AcA, 58, 153

Soszyński, I., Poleski, R., Udalski, A., et al. 2008b, AcA, 58, 163

Udalski, A., Kubiak, M., Szymański, M., et al. 1994, AcA, 44, 317

Udalski, A., Soszyński, I., Szymański, M., et al. 1999a, AcA, 49, 1

Udalski, A., Soszyński, I., Szymański, M., et al. 1999b, AcA, 49, 223

Udalski, A., Soszysńki, I., Szymański, M., et al. 1999c, AcA, 49, 437

Udalski, A., Szymański, M.K., Soszyński, I., \& Poleski, R. 2008a, AcA, 58, 69

Udalski, A., Soszyński, I., Szymański, M.K., et al. 2008b, AcA, 58, 89

Woźniak, P.R. 2000, AcA, 50, 421

Woźniak, P.R., Udalski, A., Szymański, M., et al. 2002, AcA, 52, 129

Wyrzykowski, Ł., Udalski, A., Kubiak, M., et al. 2003, AcA, 53, 1

Wyrzykowski, Ł., Udalski, A., Kubiak, M., et al. 2004, AcA, 54, 1

Żebruń, K., Soszyński, I., Woźniak, P.R., et al. 2001, AcA, 51, 317 